\title{
Experimental and numerical aspects of ship structure failure under airblast loading
}

\author{
P. Longère ${ }^{1}$, A.-G. Geffroy ${ }^{2}$, and B. Leblé ${ }^{2}$ \\ ${ }^{1}$ Université de Toulouse, ISAE/ICA (EA814), 31055 Toulouse Cedex 4, France \\ ${ }^{2}$ DCNS Ingénierie, 56311 Lorient Cedex, France
}

\begin{abstract}
This work aims at studying experimentally and reproducing numerically the failure mechanisms of a ship structure material when submitted to severe conditions of strain and strain rate. Laboratory tests and airblast experiments were accordingly carried out and a constitutive model has been built describing the salient effects of strain hardening, thermal softening, viscoplasticity and void growth induced damage. Numerical simulations were conducted considering plates subjected to various airblast loading conditions. The numerical results show clearly the influence of the damage and further fracture related model parameters, as well as the limitation of the fluid/structure interaction model used in the present work.
\end{abstract}

\section{Introduction}

This work aims at studying experimentally and reproducing numerically the failure mechanisms of a ship structure constitutive material when submitted to airblast loading. Depending on the mass of the explosive and on the distance between the structure and the explosive, one can typically observe the deflection of the plate, the nucleation of macro cracks and the phenomenon of petalling. The vulnerability oriented dimensioning of the warship hull requires accurate predictions of the three aforementioned states for airblast loaded structures in order to guarantee that the sailing functions are preserved and that the sensitive areas remain sound.

An important experimental campaign, including laboratory thermomechanical tests as well as airblast experiments allowed for characterizing the behaviour of a ship structure constitutive material under low to high strain rate conditions. The mechanisms of damage and cracking which occur during the deformation processes until the ultimate failure were identified from micrographic analyses of partially and totally fractured specimens.

Based on these experimental observations, a multisurface based elastic-thermo-viscoplastic model involving damage was built, intended to reproduce notably the behaviour transition between dense metal plasticity and microporous metal plasticity, see also [1]. Moreover, the modelling approach adopted here aims at palliating the incapacity of the usual elliptic, pressure dependent plasticity models, including GTN one (see [2], [3]), to describe void growth related damage under shear in a way preserving the classical meaning of the microporosity related damage.

The performances of the model are evaluated by comparing experimental and numerical results considering airblast loaded plates.

The airblast experimental results are presented in Sect. 2, the modelling approach is outlined in Sect. 3 and the numerical simulations are shown in Sect. 4.

\section{Airblast experiments}

The material of the present study is a ferritic-pearlitic mild steel employed as structural material for battleship hull.
Designated as DH36, it is composed of about $87 \%$ ferrite and $13 \%$ pearlite with a lamellar aspect. The microscopic analysis has revealed a negligible proportion of inclusions. The material can accordingly be considered as exempt from particles.

\subsection{Experimental results}

To qualify the material under consideration regarding explosion loading, airblast experiments were carried out. The samples were machined in the form of square thin plates. The steel plate was held down by two frames fixed to the underlying structure, see figure 1 . The explosive was spherical, hung on a post and braces. The mass of the explosive and the distance between the explosive and the plate are controlled parameters. For further details on the experimental set-up, the reader may refer to [4].

Depending on the mass of the explosive $C$, and on the distance between the plate and the explosive $\mathrm{D}$, one can typically observe three states resulting from the explosion loading : the deflection of the plate (figure 2(a)), the macrocracks incipience and growth (figure 2(b)), and the phenomenon of petalling (figure 2(c)).

\subsection{Fracture analysis}

Microscopic observations were carried out on laboratory specimens submitted to various strain magnitudes before failure and on airblast loaded plates, see figure 3. They revealed the presence of two different types of damage: quasi spherical micro-cavities occurring inside the ferritic matrix and micro-cracks developing at the ferrite-pearlite interface, see figure 4 . Both types of damage initiate at an advanced stage of the deformation process, i.e. require a certain amount of plastic strain for nucleating. In the sequel, damage will tentatively refer to both cavity growth and micro-cracking and will be described via a single internal variable, namely the volume fraction (porosity $f$ ) of both quasi-spherical cavities and crack-like voids.

\section{Constitutive relations}

In the present approach, based in part on the concepts suggested in [5], the elastic-thermo-viscoplastic metal 

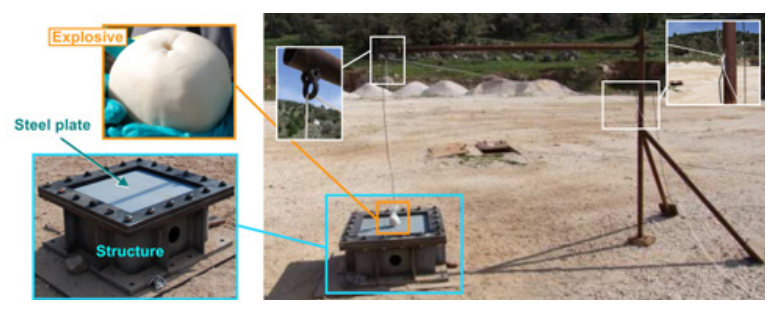

Fig. 1. Airblast experimental set-up.

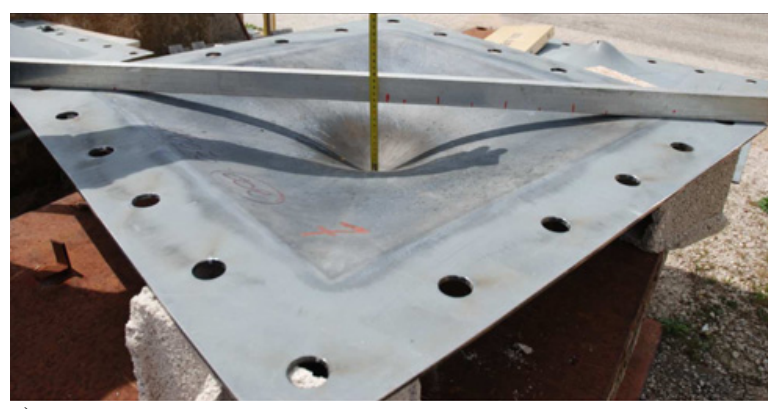

a)

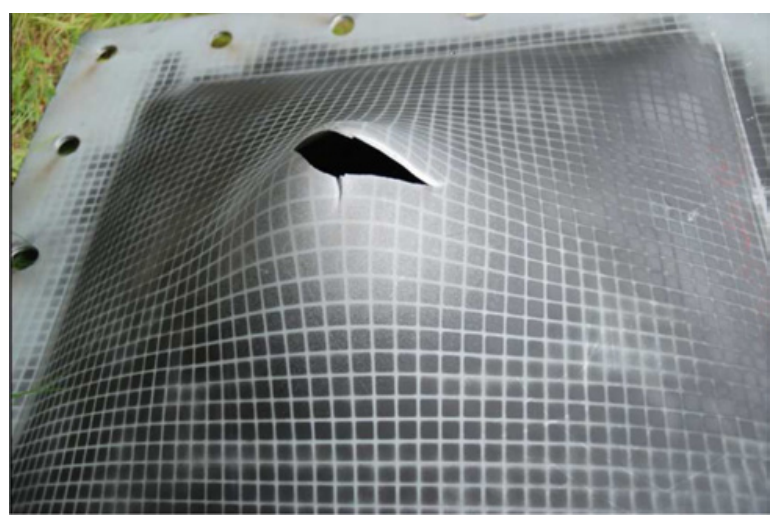

b)

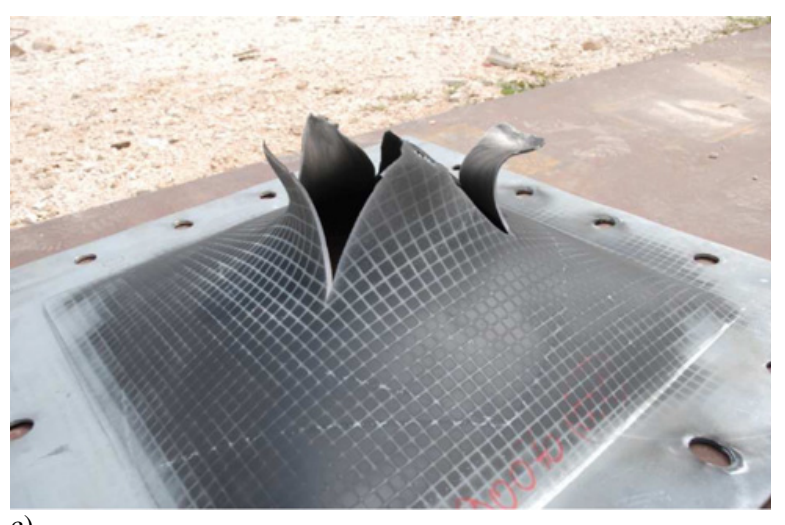

c)

Fig. 2. Experimental aspects of the plate after airblast for various loading configurations (DCNS-DGA). a) Deflection ( $\mathrm{C}=\mathrm{C}_{0}$ and $\left.\left.\mathrm{D}=\mathrm{D}_{0}\right), \mathrm{b}\right)$ Cracking start $\left(\mathrm{C}=2 \mathrm{C}_{0}\right.$ and $\left.\left.\mathrm{D}=\mathrm{D}_{0}-2.5 \mathrm{~mm}\right), \mathrm{c}\right)$ Petalling $\left(C=2 \mathrm{C}_{0}\right.$ and $\left.\mathrm{D}=\mathrm{D}_{0}-5 \mathrm{~mm}\right)$.

considered here is supposed to be initially dense, i.e. exempt from micro-voids. As soon as the conditions of plastic strain, plastic strain rate, temperature and stress triaxiality are satisfied, the material is assumed to become micro porous and its behaviour becomes pressure

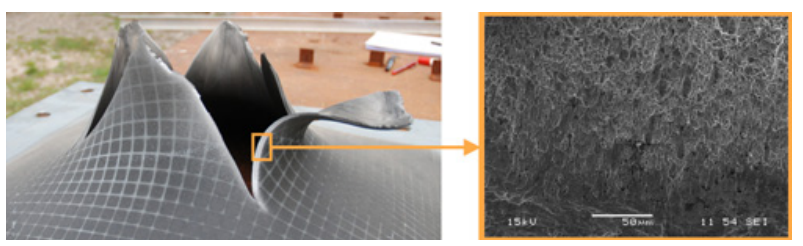

Fig. 3. Fractography of the plate after petalling revealing the presence of elongated dimples as the result of opening-shear mode of crack propagation (SEM).

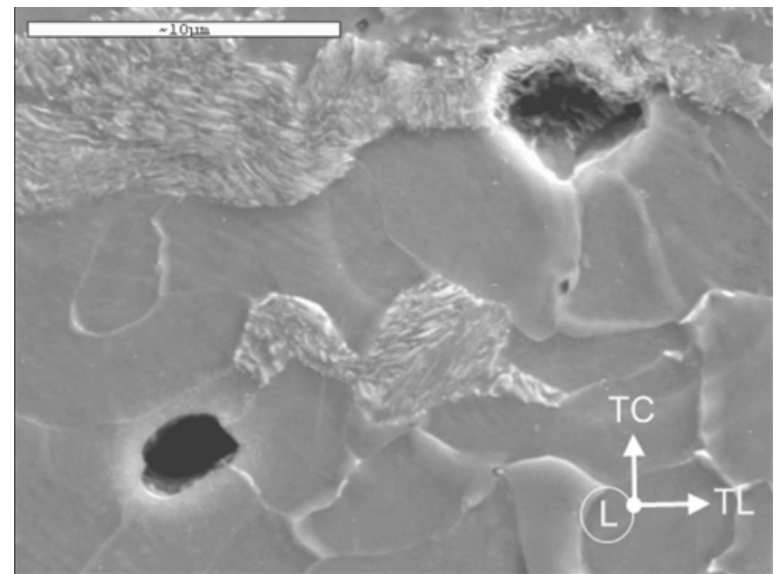

a)

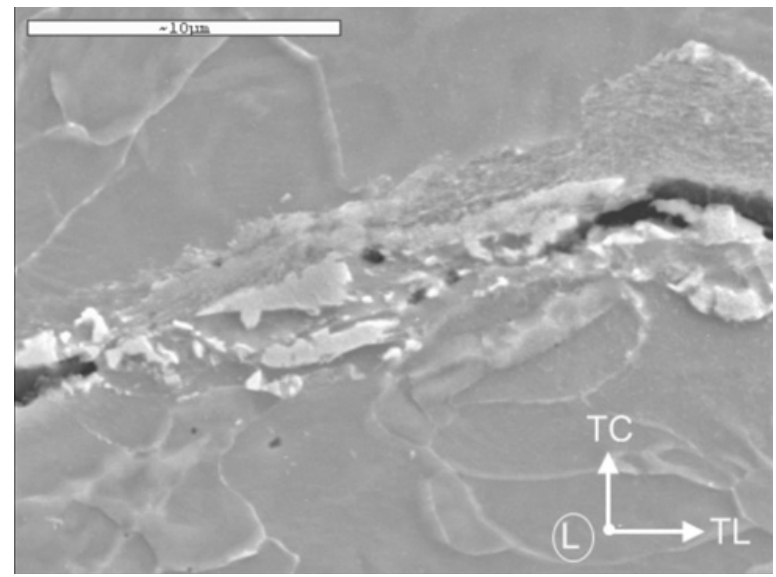

b)

Fig. 4. Types of damage as observed in specimens deformed in tension (SEM). a) Quasi spherical micro-voids at grain boundaries, b) Micro-cracks at ferrite-pearlite interphase.

dependent. The model detailed in the following aims at describing these two stages (dense state then micro porous state) in the deformation process of ductile metals. It is developed in the large deformation framework in the associated plasticity context. The internal variable procedure has been followed to describe the material behaviour in both aforementioned regimes. The damage at stake is coupled with plasticity and is supposed to occur as soon as a micro-void nucleation criterion is verified. The reader may refer to [1] for a detailed presentation of the guiding concepts of the model. 


\subsection{Constitutive relations for the dense material}

The viscoplastic yielding of the sound (undamaged) material is described via a Huber-Mises type yield function:

$$
\Phi_{0}=\tilde{\sigma}_{e q}^{2}-1=0 ; \quad \tilde{\sigma}_{e q}=\sigma_{e q} / \bar{\sigma}_{y}
$$

The yield stress $\bar{\sigma}_{y}$ in (1) includes a rate independent contribution $\sigma_{y}$ and a strain rate induced overstress $\sigma_{v p}$ :

$$
\bar{\sigma}_{y}=\sigma_{y}+\sigma_{v p}
$$

Based on the experimental campaign summarized in Sect. 2.1, the rate independent contribution $\sigma_{y}$ in (2) incorporates the combined effects of strain hardening, via a Voce type law, and thermal softening, via a power law:

$$
\begin{gathered}
\sigma_{y}=\left[R_{0}+h^{\prime}(\kappa)\right] g(T) ; h^{\prime}(\kappa)=R_{\infty}[1-\exp (-k \kappa)]^{\beta} \\
g(T)=1-\left(\frac{T}{T_{\text {melt }}}\right)^{m}
\end{gathered}
$$

where $\left(R_{0}, R_{\infty}, k, \beta\right)$ represent isotropic hardening related constants and $\left(T_{\text {melt }}, m\right)$ thermal softening related constants. With (3), the rate independent contribution $\sigma_{y}$ in (2) takes thus the form

$$
\sigma_{y}=\left\{R_{0}+R_{\infty}[1-\exp (-k \kappa)]^{\beta}\right\}\left[1-\left(\frac{T}{T_{\text {melt }}}\right)^{m}\right]
$$

The isotropic hardening force $r$ is expressed as follows

$$
r=h^{\prime}(\kappa) g(T)=R_{\infty}[1-\exp (-k \kappa)]^{\beta}\left[1-\left(\frac{T}{T_{\text {melt }}}\right)^{m}\right]
$$

The tension/compression asymmetry is assumed to be controlled by a thermally activated mechanism involving the mean stress, as it is postulated in [6]. The strain rate induced overstress $\sigma_{v p}$ in (2) is accordingly expressed in the form

$$
\sigma_{v p}=Y\left[\dot{\kappa} \exp \left(\frac{V_{a} p_{m}}{k_{B} T}\right)\right]^{1 / n}
$$

where $(Y, n)$ are viscosity related constants and $\left(V_{a}, k_{B}\right)$ behaviour asymmetry related constants.

Assuming the normality rule, the evolution laws are given by:

$$
\left\{\begin{array}{l}
\dot{\varepsilon}_{0}^{p M}=-\Lambda \frac{\partial \Phi_{0}}{\partial p_{m}}=0 \\
\dot{\varepsilon}_{0}^{p D}=\Lambda \frac{\partial \Phi_{0}}{\partial \sigma_{e q}}=2 \Lambda \frac{\tilde{\sigma}_{e q}}{\bar{\sigma}_{y}}
\end{array} ;\left\{\begin{array}{l}
\dot{\kappa}=\dot{\varepsilon}_{0}^{p D} \\
\dot{T}=\frac{\left(\sigma_{e q}-r\right)}{\rho C} \dot{\kappa}
\end{array}\right.\right.
$$

\subsection{Constitutive relations for the damaged material}

The behaviour of the micro porous material is described via a Gurson-TvergaARD-NeEdLEMAn (GTN) like model, see [2] and [3]. The approach adopted here aims at palliating the GTN model incapacity to describe void growth related damage under shear and under low and small negative triaxiality in a way preserving the classical meaning of $f$ (i.e. the microporosity related damage) and allowing for void growth under shear-dominated deformation. The proposed modification is based on the hypothesis of a kinematic mean stress mechanism which governs a shift of the yield locus centre towards negative stress triaxiality values along the increasing damage. The modified GTN yield locus is given, according to [1], in the form

$$
\begin{aligned}
\Phi_{G}= & \tilde{\sigma}_{e q}^{2}+2 q_{1} f \cosh \left[-(3 / 2) q_{2}\left(\tilde{p}_{m}+\tilde{p}_{r}\right)\right] \\
& -\left(1+q_{3} f^{2}\right)=0 ; \quad \tilde{p}_{m}=p_{m} / \bar{\sigma}_{y} ; \quad \tilde{p}_{r}=p_{r} / \bar{\sigma}_{y}
\end{aligned}
$$

Assuming the kinematic pressure $p_{r}$ as vanishing when the porosity $f$ tends to the unity, the former is given by

$$
p_{r}=b \ln \left(q_{1} f\right)
$$

where $b$ is a non-negative constant.

For the damaged material, also assumed as being standard in the thermodynamic sense, the evolution laws are given by:

$$
\begin{aligned}
& \left\{\begin{array}{l}
\dot{\varepsilon}_{G}^{p M}=-\Lambda \frac{\partial \Phi_{G}}{\partial p_{m}}=3 q_{1} q_{2} f \Lambda \frac{\sinh \left[-\frac{3}{2} q_{2}\left(\tilde{p}_{m}+\tilde{p}_{r}\right)\right]}{\bar{\sigma}_{y}} \\
\dot{\varepsilon}_{G}^{p D}=\Lambda \frac{\partial \Phi_{G}}{\partial \sigma_{e q}}=2 \Lambda \frac{\tilde{\sigma}_{e q}}{\bar{\sigma}_{y}}
\end{array}\right. \\
& \left\{\begin{array}{l}
\dot{\kappa}=\frac{\sigma_{e q} \dot{\varepsilon}_{G}^{p D}-p_{m} \dot{\varepsilon}_{G}^{p M}}{(1-f) \bar{\sigma}_{y}} \\
\dot{T}=\frac{\sigma_{e q} \dot{\varepsilon}_{G}^{p D}-p_{m} \dot{\varepsilon}_{G}^{p M}-r \dot{\kappa}}{\rho C} \\
\dot{f}=\dot{f}_{n}+\dot{f}_{g}
\end{array}\right.
\end{aligned}
$$

where $\dot{f}_{g}$ and $\dot{f}_{n}$ represents the growing rate of existing (primary) voids and the nucleating rate of secondary voids, respectively. The expression for the void growth rate is deduced from the matrix material incompressibility whereas the kinetic law for secondary nucleation is assumed to be well described by a WeIBULL type distribution function, see [7]:

$$
\left\{\begin{array}{l}
\dot{f}_{n}=B\left\langle\dot{\sigma}_{y}\right\rangle \\
\dot{f}_{g}=(1-f) \operatorname{Tr} \underline{\underline{\varepsilon}}^{p}=(1-f) \dot{\varepsilon}_{G}^{p M}
\end{array} ;\left\{\begin{array}{l}
f_{n}(0)=0 \\
f_{g}(0)=f_{0}
\end{array}\right.\right.
$$

where the quantity $B$ involves the hole nucleation function $\Phi_{I_{0}}$ and the critical stress $\sigma_{c}$ both detailed in the following subsection:

$$
B=f_{\text {sup }} \frac{p}{\sigma_{c}}\left\langle\Phi_{I_{0}}\right\rangle^{p-1} \exp \left(-\Phi_{I_{0}}^{p}\right)
$$

\subsection{Complementary damage initiation criterion}

The hole nucleation criterion describes the conditions for which a specific volume fraction of 'primary' voids $f_{0}$ instantaneously germinates. In order to ensure the instantaneous transition between dense metal plasticity and microporous metal plasticity, the hole nucleation criterion and 


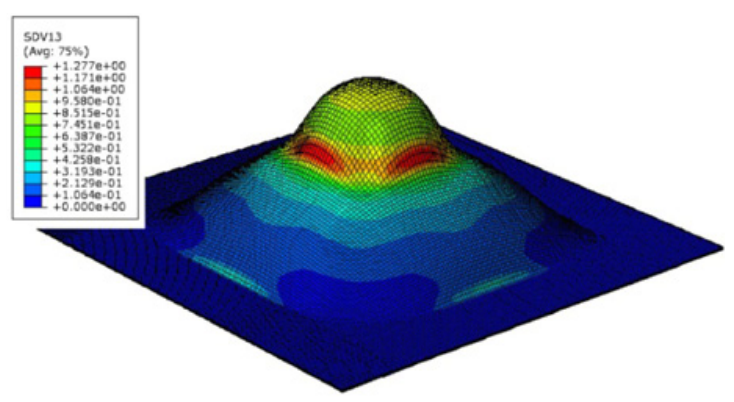

Fig. 5. $\alpha=0.65-f_{r}=0.03$.

the microporous metal potential have the similar aspect and the corresponding expressions:

$$
\begin{aligned}
\Phi_{I_{0}} & =\hat{\sigma}^{2}+2 q_{1} f_{0} \cosh \left(-(3 / 2) q_{2}\left(\hat{p}_{m}+\hat{p}_{r}\right)\right) \\
-\left(1+q_{3} f_{0}^{2}\right) & =0 ; \quad \hat{\sigma}=\sigma_{y} / \sigma_{c} ; \quad \hat{p}_{m}=p_{m} / \sigma_{c} \\
\hat{p}_{r} & =p_{r} / \sigma_{c}
\end{aligned}
$$

where $\sigma_{c}$ is a critical stress. To describe the delaying effects of temperature and strain rate the latter is assumed in the form:

$$
\sigma_{c}=\sigma_{I}+Y\left[\dot{k} \exp \left(V_{a} p_{m} /\left(k_{B} T\right)\right)\right]^{1 / n} ; \quad \sigma_{I}=\alpha\left(R_{0}+R_{\infty}\right)
$$

\section{Numerical simulations}

The model outlined in Sect. 3 has been implemented as user material in the engineering finite element computation code AвAQus ${ }^{\circledR}$. Numerical simulations of airblast tests were then performed.

The numerical integration is conducted in the GREENNAGHDI rotating frame using the classical return mapping procedure combined with the NEWTON-RAPHSON solving algorithm, see [8] for further details.

The following numerical simulations are systematically conducted using an explicit time integration scheme. The structure is composed of two parts: the steel plate, meshed using 8-node bricks with reduced integration (C3D8R) and containing five elements in the thickness; and two frames, considered as rigid bodies crimping the plate. The explosion induced fluid/structure interaction is numerically simulated via the CoNwEP card of ABAQUS ${ }^{\circledR}$ 6.10 .

Adiabatic conditions are furthermore assumed to be valid for plastic equivalent strain rate $\dot{K}$ greater than $1 \mathrm{~s}^{-1}$. In addition, failure is supposed to occur as soon as the porosity reaches the critical value $f_{r}$, leading numerically to the erosion of the concerned finite element.

\subsection{Parametric study}

We are here studying the influence of two parameters, namely the ratio $\alpha$ intervening in $(14)_{2}$ and the porosity at rupture $f_{r}$, for a given loading case.

The reference configuration is shown in figure 5 . Figures 6 and 7 show configurations with lower values

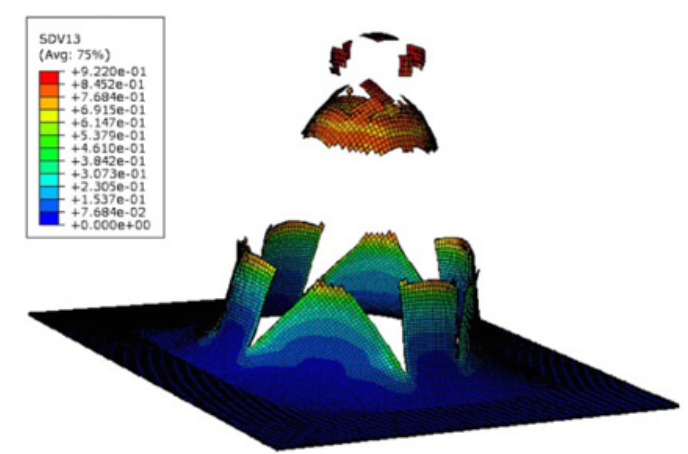

Fig. 6. $\alpha=0.60-f_{r}=0.03$.

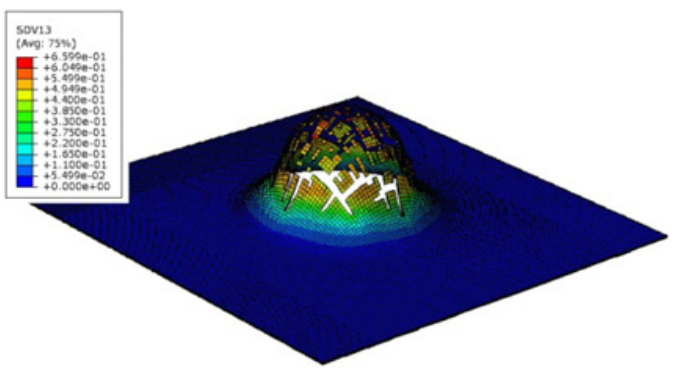

Fig. 7. $\alpha=0.55-f_{r}=0.03$.

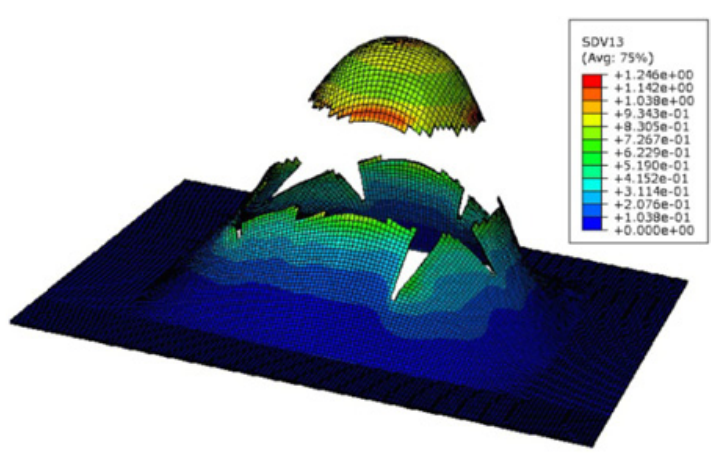

Fig. 8. $\alpha=0.65-f_{r}=0.01$.

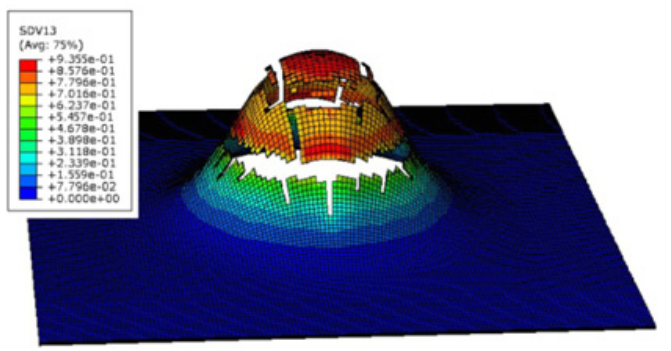

Fig. 9. $\alpha=0.65-f_{r}=0.005$.

of the ratio $\alpha$ leading to earlier local damage initiation and consequently earlier structural fragmentation. One can observe the same trend from figures 8 and 9 considering lower values of the porosity at rupture $f_{r}$.

\subsection{Comparison with experiment}

The numerical results for various loading conditions, in terms of explosive mass and plate/explosive distance, 
Table 1. Microporous model related constants for the numerical simulations

\begin{tabular}{|c|c|c|c|c|c|c|c|c|}
\hline $\boldsymbol{q}_{\mathbf{1}}$ & $\boldsymbol{q}_{\mathbf{2}}$ & $\boldsymbol{q}_{\mathbf{3}}$ & $\boldsymbol{P}$ & $\boldsymbol{f}_{\mathbf{0}}$ & $\boldsymbol{\alpha}$ & $\boldsymbol{b}(\boldsymbol{M P a})$ & $\boldsymbol{f}_{\text {sup }}$ & $\boldsymbol{f}_{\boldsymbol{r}}$ \\
\hline 1 & 1 & 1 & 2 & $10^{-4}$ & 0.55 & 115 & 0.10 & 0.03 \\
\hline
\end{tabular}

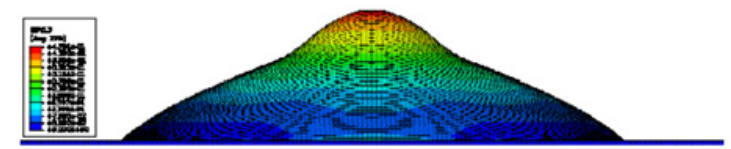

a)

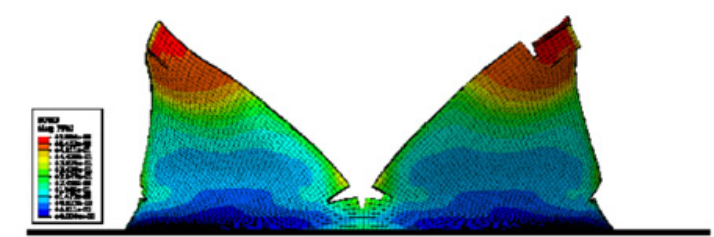

b)

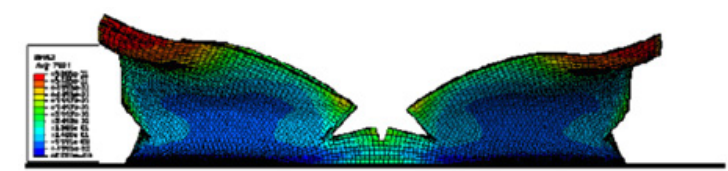

c)

Fig. 10. Experimental aspects of the plate after airblast for various loading configurations (DCNS-DGA). a) Deflection (C = $\mathrm{C}_{0}$ and $\left.\mathrm{D}=\mathrm{D}_{0}\right)$, b) Cracking start $\left(\mathrm{C}=2 \mathrm{C}_{0}\right.$ et $\left.\mathrm{D}=\mathrm{D}_{0}-3 \mathrm{~mm}\right)$, c) Petalling $\left(\mathrm{C}=2 \mathrm{C}_{0}\right.$ and $\left.\mathrm{D}=\mathrm{D}_{0}-5 \mathrm{~mm}\right)$.

corresponding to those used in the experiments are presented in figure 10 .

According to figure 10 one can note a relatively satisfying (conservative) correlation between experimental and numerical results. Though the initiation of cracking seems to be the most difficult stage to reproduce, the model with a set of constants identified appears capable to describe the petalling of the plate, see Table 1 .

It must be noted that the release of burnt gases is not possible when the ABAQUs ${ }^{\circledR}$ ConweP card, reproducing numerically approximately the fluid/structure interaction, is used. This implies that the explosion induced pressure keeps on being applied on the plate surface all along the loading duration, provoking a petal reversal more important than in reality. Consequently, for a better description of the airblast induced failure of the plate, a more accurate reproduction of the fluid-structure interaction is needed.

\section{Concluding remarks}

An important experimental campaign, including laboratory thermomechanical tests as well as airblast experiments allowed for characterizing the behaviour of a ship structure constitutive material. The mechanisms of damage and cracking which occur during the deformation processes until the ultimate failure were identified from micrographic analyses of partially and totally fractured specimens.

Some experimental results were used to build a multisurface based constitutive model able to describe the transition between dense metal plasticity and microporous metal plasticity. The criterion of damage initiation accounts for the accelerating effects of stress triaxiality and for the delaying effects of temperature and strain rate. A modification of the GTN model has also been proposed permitting to describe the cavity growth under shear loading.

After identifying a set of material constants from standard experiments, the model was evaluated considering numerical experiments of explosion loaded plates. The corresponding results are encouraging when compared qualitatively with experimental data. A better description of the fluid/structure interaction, numerically reproduced in the present work via the ABAQUS ${ }^{\circledR}$ ConweP card, could allow for improving the predictive capacity of the numerical model.

\section{References}

1. P. Longère, A.-G. Geffroy, B. Leble, A. Dragon, Int. J. Dam. Mech., DOI: 10.1177/1056789511427472, (2011).

2. A. L. Gurson, J. Eng. Mat. Tech., 99 (1977)

3. V. Tvergaard, A. Needleman, Acta metall, 32 (1984)

4. A.-G. Geffroy, P. Longère, B. Leble,, Eng. Fail. Anal., 18-2 (2011)

5. A. Dragon, Eng. Fract. Mech., 21-4 (1985)

6. S. Graff, S. Forest, S. Strudel, J.-L. Prioul, P. Pilvin, J.-L. Bechade, Mat. Sci. Eng., A 387-389 (2004)

7. A. Molinari, T. W. Wright, J. Mech. Phys. Solids, 53 (2005)

8. N. Aravas, Int. J. Num. Meth. Eng., 4 (1987) 